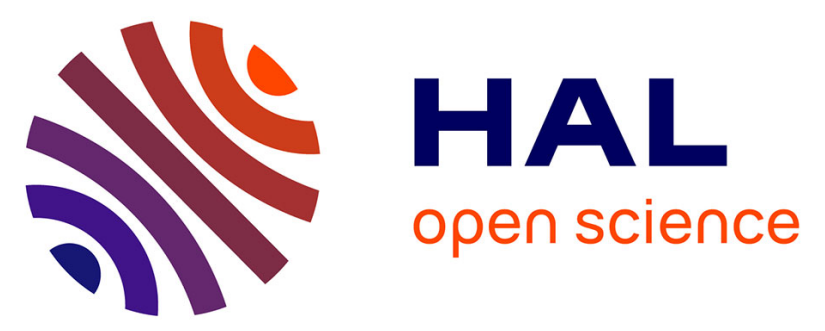

\title{
Performance of individual vs. group sampling for inferring dispersal under isolation-by-distance
}

Natacha Luximon, Eric Petit, Thomas Broquet

\section{To cite this version:}

Natacha Luximon, Eric Petit, Thomas Broquet. Performance of individual vs. group sampling for inferring dispersal under isolation-by-distance. Molecular Ecology Resources, 2014, 14 (4), pp.745-752. 10.1111/1755-0998.12224. hal-01062314

HAL Id: hal-01062314

https://hal-univ-rennes1.archives-ouvertes.fr/hal-01062314

Submitted on 7 Jan 2015

HAL is a multi-disciplinary open access archive for the deposit and dissemination of scientific research documents, whether they are published or not. The documents may come from teaching and research institutions in France or abroad, or from public or private research centers.
L'archive ouverte pluridisciplinaire HAL, est destinée au dépôt et à la diffusion de documents scientifiques de niveau recherche, publiés ou non, émanant des établissements d'enseignement et de recherche français ou étrangers, des laboratoires publics ou privés. 
Performance of individual vs group sampling for inferring dispersal under isolation by distance

Natacha Luximon ${ }^{1}$, Eric J. Petit ${ }^{2}$, Thomas Broquet ${ }^{3}$

4

1- Department of Ecology and Evolution, University of Lausanne, $\mathrm{CH}-1015$ Lausanne, Switzerland.

6 2- Univ Rennes 1, CNRS, UMR 6553 ECOBIO, Station Biologique, F-35380 Paimpont, France

3- Team Diversity and connectivity of coastal marine landscapes, UMR 7144, Station Biologique de 8 Roscoff, CNRS \& Univ Paris 06.

10

Keywords

12 Dispersal inference, gene flow, individual-based simulations, dispersal kernel, IBD

14

Corresponding author

Thomas Broquet, Team Div\&Co, UMR 7144, Station Biologique de Roscoff, Place Georges Teissier, 29680 Roscoff, France, phone number: +33 298292 312, Email: thomas.broquet@sb-roscoff.fr

18

20

Running title

22 Group vs individual IBD analysis 
Models of isolation-by-distance formalize the effects of genetic drift and gene flow in a spatial context where gene dispersal is spatially limited. These models have been used to show that, at an appropriate spatial scale, dispersal parameters can be inferred from the regression of genetic differentiation against geographic distance between sampling locations. This approach is compelling because it is relatively simple and robust, and has rather low sampling requirements. In continuous populations, dispersal can be inferred from isolation-by-distance patterns using either individuals or groups as sampling units. Intrigued by empirical findings where individual samples seemed to provide more power, we used simulations to compare the performances of the two methods in a range of situations with different dispersal distributions. We found that sampling individuals provides more power in a range of dispersal conditions that is narrow but fits many realistic situations. These situations were characterized not only by the general steepness of isolation-bydistance but also by the intrinsic shape of the dispersal kernel. The performances of the two approaches are otherwise similar, suggesting that the choice of a sampling unit is globally less important than other settings such as a study's spatial scale.

Introduction

Genetic data can inform us about dispersal patterns. But that information can be obtained only when a number of biological and methodological conditions are fulfilled. At one end of a methodological continuum, the direct identification of dispersal events (e.g. using population or parentage assignment) can provide detailed and accurate dispersal data. But because of its reliance on intensive sampling, this approach is constrained in terms of study systems, time frame, and study area. At the other end, indirect estimates of migration rates obtained from measurements of spatial genetic structure and demogenetic models depend critically on models' refinement and assumptions 
(Marko \& Hart 2011; Whitlock \& McCauley 1999). A sustained interest in this field of research has produced a wealth of alternative potential solutions for inferring dispersal (e.g. reviewed in Broquet \& Petit 2009 and other references therein), but finding a good fit between biological settings and methodological options is rarely obvious.

Inferring dispersal from isolation-by-distance (IBD) patterns is one approach that seems to stand out by its (relatively) wide applicability. The dynamics of genetic variation in populations along a gradient of spatial proximity were first formalized by Wright (1943), Malécot (1949), and Kimura \& Weiss (1964). These and following IBD theoretical developments have set ground for several inference methods that aim at estimating dispersal from genetic data (reviewed in Guillot et al. 2009). We focus here on the method proposed by Rousset $(1997,2000)$, which uses a regression of genetic distances on geographic distances among pairs of samples to infer the product $D \sigma^{2}$, where $D$ is the effective density and $\sigma^{2}$ is the mean squared parent-offspring distance. If $D$ can be independently estimated then $\sigma^{2}$ gives a synthetic descriptor of dispersal that can be compared across populations or species (e.g. Pinsky et al. 2010; see also Vekemans \& Hardy 2004 using a related approach), and possibly compared with field-based estimates (e.g. Watts et al. 2007). The product $D \sigma^{2}$ itself is also of interest as it informs us on the increase of differentiation with distance. This approach is not free from drawbacks. Most importantly, the parameter $\sigma$ is not intuitive (see discussions in Broquet \& Petit 2009; Rousset 2004; Sumner et al. 2001), some preliminary knowledge of dispersal scale is needed to set an appropriate study scale, and data interpretation requires some understanding of the effect of departure from mutation-migration-drift equilibrium. But the method's robustness or behavior has been assessed in various aspects (e.g. Broquet et al. 2006b; Leblois et al. 2003; Leblois et al. 2004; Vekemans \& Hardy 2004; Watts et al. 2007), and it relies on manageable sampling requirements. Accordingly, interpretations of isolation-by-distance patterns are frequent in the literature, including several estimations of the dispersal parameter $\sigma$ (reviewed in Table S1, supplementary material. See also Fig. 1). 
Rousset proposed to calculate distances between individuals in a continuous population (Rousset 2000) or between groups of individuals (either because the population under study is subdivided into discrete units, or because discrete groups of individuals were sampled from an otherwise continuous population; Rousset 1997, 2000). Hereafter we will use the words "individual" and "group" to refer to the sampling unit of each approach. The two methods are based on the same theoretical background (detailed in Rousset 2004) and aim at estimating exactly the same quantity. Importantly, the two methods should be used at the same spatial scale, considering samples at distances not greater than ca. $0.56 \sigma / \sqrt{2 \mu}$, where $\mu$ is the mutation rate of the loci considered (Rousset 2004). Because the regression method based upon groups can be applied in a continuous population, some empirical case studies compared the results provided by the two methods with the same species in the same population (Broquet et al. 2006a; Suni \& Gordon 2010; Watts et al. 2007). These studies repeatedly found that the group approach has less power, in some cases to the point that only the individual approach could be used to infer $\sigma$. The correlation of genetic and geographic distances is tested using Mantel's test, which is not particularly powerful (Legendre \& Fortin 2010), and the number of pairwise comparisons is easily two orders of magnitude greater when using individuals as sampling units. The difference in power observed in case studies could thus be due simply to the number of data points, giving an advantage to individuals as sampling units. On the other hand, individual-based genetic distances may suffer from more sampling variance and more variable effect of genetic drift than group-based statistics. Differences in power remain to be investigated and complemented with results for the precision, bias, and coverage of confidence intervals obtained with each approach. The performances of IBD-based dispersal inference have been thoroughly evaluated in simulation studies that used individuals as sampling units (Leblois et al. 2003; Leblois et al. 2004). However, individual- and group-based sampling schemes have not yet been compared to one another in controlled conditions. Such a comparison could be useful for planning field studies and for interpreting empirical patterns, particularly in situations where samples are not easily collected individually. Such comparisons are also timely because of the 
growing interest in using pooled samples (mixtures of individuals) that develops in parallel with modern sequencing protocols (Davey et al. 2011; Futschik \& Schlotterer 2010; Gautier et al. 2013). Our objective is to determine whether there is an advantage in using one or the other method in situations where the two methods could be applied.

Methods

Using IBDSim (Leblois et al. 2009) we simulated a continuous population composed by a square grid of $110 \times 110$ units with one diploid individual per node. Each individual was characterized by a multilocus genotype made of 10 microsatellites. IBDSim simulates the demography (coalescence and dispersal) backwards in time before adding in mutations. The life-cycle is as follows: i) gamete production and death of adults. ii) gamete mutation following a generalized stepwise model with rate $\mu=5 \times 10^{-4}$ as described in Leblois et al. (2004) with a maximum number of alleles set to 100 per locus (a value large enough to be uninfluential here). iii) gamete dispersal according to a predefined distribution of dispersal distances (see below). iv) constitution of diploid individuals. v) regulation of the population to $n=1$ individual per node.

We defined 36 simulation scenarios (Table S1) differing only in dispersal conditions. Dispersal distances followed a truncated Pareto distribution, where the probability of dispersing $k$ steps in each dimension is given by $f_{k}=M / k^{n}$ for $k \leq k_{\text {max }}$, as discussed by Rousset (2000). We varied $M$ (total dispersal rate in one dimension), $n$ (a parameter that controls the shape of the distribution) and $k_{\max }$ (maximum dispersal distance) to obtain a range of dispersal situations with simulated $\sigma$ values $\left(\sigma_{\text {sim }}^{2}\right.$, range $\left.1.12-47.01\right)$ comparable to that estimated from empirical case studies $\left(\sigma_{\text {est }}^{2}\right.$ : we found estimates for 62 plant and animal species, Fig. 1 and Table S1). Simulations thus differed in the values taken by $\sigma^{2}$ (giving the strength of IBD) but also in the nature of the dispersal kernels characterized by $M, n$ and $k_{\max }$ - that yielded these values. 
Dispersal inference under IBD should consider samples at distances smaller than ca.

$0.56 \sigma / \sqrt{2 \mu}$ (Rousset 2004), which is approximately equal to $18 \sigma$ given our mutation rate. The total size of the simulated population $(110 \times 110)$ was large enough to contain the optimal sampling design for any simulation scenario with some extra space to limit edge effects. At grid edges we used "absorbing" boundaries in IBDSim whereby "the probability mass of going outside the lattice is equally shared on all other movements inside the lattice" (as defined by R. Leblois in IBDSim user manual). The total simulated population was kept constant but samples were taken from within a smaller area and defined as a square of side length $13 \sigma$ (that is, with diagonal $\approx 18 \sigma$, Fig. 2 ). A different sampling grid was thus potentially associated with each simulation scenario.

To test for IBD and infer $\sigma^{2}$ we randomly sampled 99 individuals and 11 disjoint clusters of 9 individuals from within the defined sampling grid (Fig. 2). These samples were analyzed in Genepop V4.0 (Rousset 2008) using the estimator $\hat{a}$ for pairwise genetic distances among individuals (Rousset $2000)$ and $F_{S T} /\left(1-F_{S T}\right)$ for groups (Rousset 1997). The mean genetic distance among pairs of samples and the global $F_{\mathrm{ST}}$ are shown in Table S2 and Fig. S1. The slope $(b)$ of the regression of pairwise genetic distances and In-transformed geographic distances among samples (individuals or groups) was used to infer $\sigma^{2}$ from the relationship $b=1 /\left(4 D \pi \sigma^{2}\right)$ with $D=1$. We also recorded approximate 95\% confidence intervals calculated using the ABC procedure implemented in Genepop (Leblois et al. 2003; Rousset 2008; Watts et al. 2007). Each simulation was replicated 200 times (a number large enough to capture most of the variance across replicates, data not shown), giving $36 \times 200=7200$ simulations overall.

The power of the regression method based upon groups and individuals was calculated for the 14436 simulation conditions as the proportion of replicates yielding a significant Mantel test (using 10 000 permutations and a significance threshold $\alpha=5 \%)$. The relative error was estimated as $\left(\sigma_{\text {est }}^{2}-\sigma_{\text {sim }}^{2}\right) / \sigma_{\text {sim }}^{2}$ for each replicate, and we defined the bias and the precision of $\sigma^{2}$ estimates as the median and the dispersion of the relative error, respectively. Finally, the coverage was defined 
as the proportion of replicates where $\sigma_{\text {sim }}^{2}$ was included within the confidence interval of $\sigma_{\text {est }}^{2}$. These statistics were computed using only the replicates where a significant IBD was detected (5045 and 5357 replicates for the group and individual methods), because $\sigma^{2}$ would not be inferred from a dataset otherwise. between methods, and ii) the effect of parameters $M, n$, and $k_{\max }$ on the power, bias, and coverage of each method (with adequate transformation of data or binomial error structure when necessary). We included $\sigma_{\text {sim }}^{2}$ as an explanatory variable in these models because it is directly linked to the strength of IBD and thus should be a primary determinant of a method's performances. All results reported in the main text are thus independent of the value taken by $\sigma_{\text {sim }}^{2}$. To control for the fact that different simulation conditions were associated with different sampling grids (i.e. different spatial scales), we also included the median of the Euclidean distances among pairs of samples as an explanatory variable. Finally, the models were of the form $<$ response $\sim \sigma_{\text {sim }}^{2}+$ Med.dist + Method $>$ when we compared the two methods (Med. dist is the median of distances among samples) and of the form $<$ response $\sim \sigma_{\text {sim }}^{2}+$ Med. dist $+n \times M \times k_{\max }>$ when we assessed the effects of simulation parameters, where response was either power, bias, or coverage.

Results

The power of the two methods, measured as the proportion of replicates yielding a significant Mantel test, dropped from $100 \%$ to ca. $20 \%$ in our two most extreme situations in terms of simulated dispersal (Fig. 3a, $\sigma_{\text {sim }}^{2}=1.12$, and Fig. 3i, $\sigma_{\text {sim }}^{2}=47.05$ ). However, the group approach lost power at an earlier stage as the strength of IBD decreased (Figs. 3d-e). Interestingly, this effect was primarily due to the shape parameter $n$ (e.g. Figs. 3a,d,g), which had a significant effect independently of the value taken by $\sigma_{\text {sim }}^{2}(p<0.001)$. When $n$ was large (meaning that long-distance 
dispersal was rare, first row in Fig. 3 ) the two methods performed well, and $M$ and $k_{\max }$ took no effect. With low $n$ the effect of $k_{\max }$ became critical (last row of Fig. 3 ) but the two methods were equally affected. When $n$ was intermediate (middle row of Fig. 3) the group approach was more strongly affected than the individual approach by an increase in $k_{\max }$ (e.g. in Fig. $3 \mathrm{~d}$ the power decreased from $100 \%$ to $80 \%$ for the individual approach vs $60 \%$ for the group approach when $k_{\max }$ was increased from 10 to 50). These results convey the following information: i) the two methods have comparable power except in a restricted set of conditions, ii) those dispersal conditions where individuals outperformed groups resulted in $\sigma_{\text {sim }}^{2}$ in [3.89-11.83], a range of values that fits well empirical estimates from real case studies (Fig. 1), including one study where IBD was detected with individuals only (Broquet et al. 2006a), and iii) these conditions are not determined solely by $\sigma^{2}$ but also by the shape of the underlying dispersal kernel (e.g. the range of $\sigma_{\text {sim }}^{2}$ mentioned above is also spanned by simulations $25-32$, and yet with these simulations the two methods have nearly identical power, Fig. 3c\&f).

Besides power, we looked at the bias and the precision of $\sigma^{2}$ estimates with the median and the dispersion of the relative error, respectively. We found that the two methods generally underestimated the true $\sigma^{2}$ by a small proportion (Fig. 4) and that this bias was slightly more pronounced with the individual approach $(-15 \%$ and $-9 \%$ for individuals and groups overall simulations, $\mathrm{p}<0.001)$. This slight underestimation is in agreement with simulation results obtained by Leblois et al. $(2003 ; 2004)$ when the sampling design was not too far from theoretical optimum (e.g. simulations 1 and 2 in Table 2 of Leblois et al. 2003, note that the bias is calculated for the regression slope). In agreement with results for the power, the two methods showed decreasing performance (increasing bias) with decreasing IBD strength (down to ca. $-60 \%$ with $\sigma_{\text {sim }}^{2}=47.05$, Fig. $1944 \mathrm{i})$. Irrespective of $\sigma_{\text {sim }}^{2}$, the bias also appeared to be influenced by the shape of the dispersal kernel, and particularly by parameter $k_{\max }(\mathrm{p}<0.001)$. Increasing $k_{\max }$ resulted in deeper negative bias whatever the values taken by the other parameters. Surprisingly, the precision of estimates followed an opposite trend (Fig. 4): the dispersion of estimated values around the median was greatest when 
IBD was strong, and this effect was particularly visible for small values of $k_{\max }$ (left box-plot of each panel in figure 4). As a result, the situations where the bias was minor were generally not favorable in terms of precision. This observation is valid for the two methods, which showed no systematic difference in precision. Yet a difference can be noted regarding the replicates producing the worst estimates. Overall simulations with significant IBD, 17 such replicates (out of 10402 ) produced estimates with a relative error larger than $150 \%$ (Fig. 4). These cases were all characterized by a near-zero slope estimate, yielding large relative errors. Interestingly, only 3 such cases were produced by the individual approach.

Finally, we did not find any difference in coverage between methods ( $p>0.05)$ : the proportion of replicates where the $95 \%$ confidence interval of the estimate $\left(\sigma_{\text {est }}^{2}\right)$ included the true value $\left(\sigma_{\text {sim }}^{2}\right)$ amounted to $86 \%$ using groups and $85 \%$ using individuals (Fig. S2). In the specific cases where a difference in coverage was visible the method with the best coverage also appeared to have larger confidence intervals (data not shown). Note that the coverage values reported here for each method independently may be overestimated, because the $A B C$ procedure used to approximate $95 \%$

212 confidence intervals generally underestimates the upper bound for $\sigma_{\text {est }}^{2}$ (Leblois et al. 2003).

Our simulations were parameterized so that the product $D \sigma^{2}$ fits real situations where IBD patterns 216 had been analyzed (Fig. 1 and Table S1). Yet the conditions of dispersal inference varied widely between simulations for the following reason: the number of samples was kept constant across simulations (99 genotypes) while the sampling scale was set with respect to $\sigma_{s i m}^{2}$ in order to fit the methods' requirements (distance between samples $<0.56 \sigma_{\text {sim }} / \sqrt{2 \mu}$ ). It means that the density of the sampling effort decreased with increasing $\sigma_{\text {sim }}^{2}$, giving us a range of conditions where the inference of dispersal went from being very favored (when $\sigma_{\text {sim }}^{2}$ is small and IBD is steep with 
respect to the sampling scale) to very limited (with larger $\sigma_{\text {sim }}^{2}$ ). This variation allowed us to explore potential differences between the individual-based and group-based methods.

We find that there is only a small region of parameters where individual sampling outperformed group sampling, and this advantage bears upon power only (we found no sizeable differences in accuracy, precision, and coverage between the two approaches). However, we note that intermediate situations, where the power of the individual-based regression approach was greater than that of the group approach, appeared to cover the range of situations most commonly encountered in natural situations, at least in terms of $D \sigma^{2}$ (Fig. 1, exactly half of the reviewed empirical estimates fall in the $D \sigma^{2}$ region where the individual approach can outperform the group approach, depending on dispersal distributions).

Interestingly, the difference in performances between methods is due to particular conditions of $\sigma_{\text {sim }}^{2}$ but also to the shape of the dispersal kernel (decreasing $n$ significantly affected the difference in power between methods independently of $\sigma_{\text {sim }}^{2}$, see Figs. 3a,d,g). Based upon empirical finding for a forest-dwelling mammal, the American marten, we had the intuition that dispersal kernels characterized by a fat tail of long distance events could affect IBD patterns based upon groups more than individuals (Broquet et al. 2006a). But this idea is not supported by theory

238 (Rousset 2000), and our simulation results suggest that although there really is some effect of the shape of the dispersal kernel on the power of the two methods, it is not particularly due to long distance dispersal.

We also found a slightly reduced risk to get extremely biased estimates with the individual approach (considering those few estimates that were off by $150 \%$ or more, most came from group sampling). Furthermore, the accuracy of each method increased with the proportion of simulation replicates 244 where the two methods yielded a significant IBD pattern. This means that when one method yields a significant result but the other one does not then there is a higher risk of bias using either approach. In other words, with adequate datasets that fulfill the methods' assumptions, the power difference 
that may favor the individual-based approach occurs in situations where the risk of bias is anyways higher on average.

There are a number of relevant issues that were not considered here, such as the effects of 250 spatial and temporal heterogeneity in population density on the relative performances of each approach (the density was set to 1 individual per node in all our simulations, see Leblois et al. 2003;

252 Leblois et al. 2004 for different conditions with individual sampling). Whether or not such factors could interact with our findings is difficult to tackle, even using simplified simulations. Moreover, all our simulations fulfilled one critical assumption of IBD-based inferences (Rousset 1997, 2000): migration and drift are stable in space and time, and the pattern of increase of differentiation with geographic distance has reached equilibrium. The results presented here do not apply to other situations, which are irrelevant for inferring dispersal from IBD slopes, though the method seems robust to some disequilibrium situations (Leblois et al. 2004). Finally, we did not explore the effect of the number of samples (e.g. the number and the composition of groups). We chose to use rather small groups to get conservative results with the group approach, and because it is difficult to design simulation conditions that harmonize the requirements for sampling scale, useful $\sigma_{\text {sim }}^{2}$, and simulation and analysis time. In a pilot study we found nonetheless that increasing the total number of individuals sampled for each method benefited more to the group approach (data not shown). populations there is only a slight advantage in using individuals as the sampling unit. Other considerations might thus be more important, such as the spatial scaling of IBD studies. As shown by previous work, the study scale should be large enough so that dispersal becomes spatially limited 268 (unlike in the island model, which may apply at a shorter scale, e.g. see Kerth \& Petit 2005), and, more critically, local enough so that the effect of gene flow does not faint out in front of mutation and is not blurred by non-equilibrated patterns (such as signatures of past colonization, e.g. Austin et al. 2004). Hence priority should be given to identifying the right study scale and choose the 
sampling unit based upon the spatial distribution of individuals (Rousset 2000) and sampling possibilities rather than intrinsic properties of the methods. We emphasize that our conclusions about the detailed effect of dispersal parameters should not be extrapolated without caution to systems more complex than the simulations described here. But one robust result of this study is that in any case the choice of adequate spatial and temporal scales seems much more important than the sampling unit in continuously distributed populations.

Acknowledgements

We thank Raphaël Leblois for insightful discussions and comments, and for answering our questions regarding the software IBDSim. We are grateful to Glenn Yannic, Editor O. Gaggiotti and two anonymous reviewers for their constructive comments on the manuscript. We also thank Christophe Caron for his help with using the computer cluster in the biological station of Roscoff. TB was supported by the "Marine Aliens and Climate Change" program funded by AXA Research Funds.

Austin JD, Lougheed SC, Boag PT (2004) Controlling for the effects of history and nonequilibrium conditions in gene flow estimates in northern bullfrog (Rana catesbeiana) populations. Genetics 168, 1491-1506.

Broquet T, Johnson CA, Petit E, et al. (2006a) Dispersal and genetic structure in the American marten, Martes americana. Molecular Ecology 15, 1689-1697.

292 Broquet T, Petit E (2009) Molecular estimation of dispersal for ecology and population genetics. Annual Review of Ecology, Evolution and Systematics 40, 193-216.

294 Broquet T, Ray N, Petit E, Fryxell JM, Burel F (2006b) Genetic isolation by distance and landscape connectivity in the American marten (Martes americana). Landscape Ecology 21, 877-889. 
Davey JW, Hohenlohe PA, Etter PD, et al. (2011) Genome-wide genetic marker discovery and genotyping using next-generation sequencing. Nature Reviews Genetics 12, 499-510.

298

300

302

Futschik A, Schlotterer C (2010) The Next Generation of Molecular Markers From Massively Parallel Sequencing of Pooled DNA Samples. Genetics 186, 207-218.

Gautier M, Foucaud J, Gharbi K, et al. (2013) Estimation of population allele frequencies from nextgeneration sequencing data: pool- versus individual-based genotyping. Molecular Ecology 22, 3766-3779.

Guillot G, Leblois R, Coulon A, Frantz AC (2009) Statistical methods in spatial genetics. Molecular Ecology 18, 4734-4756.

Kerth G, Petit E (2005) Colonization and dispersal in a social species, the Bechstein's bat (Myotis bechsteinii). Molecular Ecology 14, 3943-3950.

Kimura M, Weiss GH (1964) The stepping stone model of population structure and the decrease of genetic correlation with distance. Genetics 49, 561-576.

Leblois R, Estoup A, Rousset F (2003) Influence of mutational and sampling factors on the estimation of demographic parameters in a "continuous" population under isolation by distance. Molecular Biology and Evolution 20, 491-502.

Leblois R, Estoup A, Rousset F (2009) IBDSim: a computer program to simulate genotypic data under isolation by distance. Molecular Ecology Resources 9, 107-109.

Leblois R, Rousset F, Estoup A (2004) Influence of spatial and temporal heterogeneities on the estimation of demographic parameters in a continuous population using individual microsatellite data. Genetics 166, 1081-1092.

Legendre P, Fortin MJ (2010) Comparison of the Mantel test and alternative approaches for detecting complex multivariate relationships in the spatial analysis of genetic data. Molecular Ecology Resources 10, 831-844.

Malécot G (1949) Les processus stochastiques en génétique de population. Publication de I'Institut de statistiques de l'Université de Paris I: Fasc 3, 1-16. 
Marko PB, Hart MW (2011) The complex analytical landscape of gene flow inference. Trends in Ecology \& Evolution 26, 448-456.

Pinsky ML, Montes Jr. HR, Palumbi SR (2010) Using isolation by distance and effective density to estimated dispersal scales in anemonefish. Evolution 64, 2688-2700. by distance. Genetics $145,1219-1228$.

Rousset F (2000) Genetic differentiation between individuals. Journal of Evolutionary Biology 13, 58-

62.

Rousset F (2004) Genetic structure and selection in subdivided populations Princeton University Press, Princeton.

Rousset F (2008) GENEPOP ' 007: a complete re-implementation of the GENEPOP software for Windows and Linux. Molecular Ecology Resources 8, 103-106.

Sumner J, Rousset F, Estoup A, Moritz C (2001) Neighbourhood size, dispersal and density estimates in the prickly forest skink (Gnypetoscincus queenslandiae) using individual genetic and demographic methods. Molecular Ecology 10, 1917-1927.

Suni SS, Gordon DM (2010) Fine-scale genetic structure and dispersal distance in the harvester ant Pogonomyrmex barbatus. Heredity 104, 168-173.

Vekemans X, Hardy O (2004) New insights from fine-scale genetic structure analyses in plant populations. Molecular Ecology 13, 921-935.

Watts PC, Rousset F, Saccheri IJ, et al. (2007) Compatible genetic and ecological estimates of dispersal rates in insect (Coenagrion mercuriale : Odonata : Zygoptera) populations: analysis of 'neighbourhood size' using a more precise estimator. Molecular Ecology 16, 737-751.

344 Whitlock MC, McCauley DE (1999) Indirect measures of gene flow and migration : $F_{S T \neq} 1 /(4 \mathrm{Nm}+1)$. Heredity 82, 117-125. 
Data accessibility

350 Three types of data are available on Dryad (entry doi:10.5061/dryad.mq8n5): i) parameter files for each simulation (IBDSim settings), ii) command lines for the batch analysis of simulation outputs in

352 Genepop, and iii) summaries of the main statistics produced by Genepop for all simulations and replicates that we used here to compare the performances of individual-based vs group-based 354 inferences.

356 Author contributions

N.L. performed the simulations and analyses. E.P. and T.B. designed the research, performed the analyses, and wrote the paper. 
Figure 1 - Comparison of the frequency distribution of empirical (plotted as positive frequencies, in

362 grey) and simulated (represented by negative frequencies, in white) values of the product $D \sigma^{2}$. Empirical values were obtained from a literature survey of significant IBD patterns for animal and 364 plant case studies that investigated two-dimensional spatial genetic structure (the product $D \sigma^{2}$ has a different scale in 1D studies, Rousset 1997). These values were either taken directly from the papers, 366 or calculated from related statistics, such as Sp (Vekemans \& Hardy 2004). When more than one value was available for a given species in a specific paper, only one was retained for drawing the histogram. Most empirical values included in this comparison are taken from the review by Vekemans and Hardy (2004), completed with results from additional papers reviewed in our Table S1 (supplementary material). Vertical lines show the lower and upper limits of the region in which individual-based analyses can outperform group-based analyses (see results). Note that the $\mathrm{x}$ axis is log-scaled for a better visualization of the distributions.

374 Figure $2-$ Principle of the sampling design. The actual simulations used a $110 \times 110$ grid, large enough to contain a square of side length $13 \sigma$ for any of the conditions listed in Table S1. Ninety-nine individuals or 11 groups of 9 individuals were randomly sampled from within this grid to infer $\sigma^{2}$ using isolation-by-distance patterns.

Figure 3 - Power of Mantel test in detecting a correlation between genetic and geographic distances among pairs of individuals (dashed black lines) or groups (solid grey lines) sampled from simulated datasets. The power was calculated as the proportion of replicates ( $n=200$ replicates per simulation scenario) where a significant correlation was detected. Simulations differ in the distribution of dispersal distances (parameters $M, n$, and $k_{\max }$ of a truncated Pareto distribution). 
384 Figure 4 -Relative error in $\sigma^{2}$ estimated from the regression of genetic- vs geographic distances between pairs of individuals (white boxes) or groups (grey). Data from 200 replicates per simulation are shown (simulation conditions as in Figure 3). The solid line in each box shows the median of the error distribution, the box shows the $25 \%$ and $75 \%$ quantiles, and the whiskers show the full range of the errors. In cases where the whiskers extend beyond the plotting region, 1 to 3 replicates (out of 200) had a relative error greater than 1.5 and are not shown here. 
Fig. 1

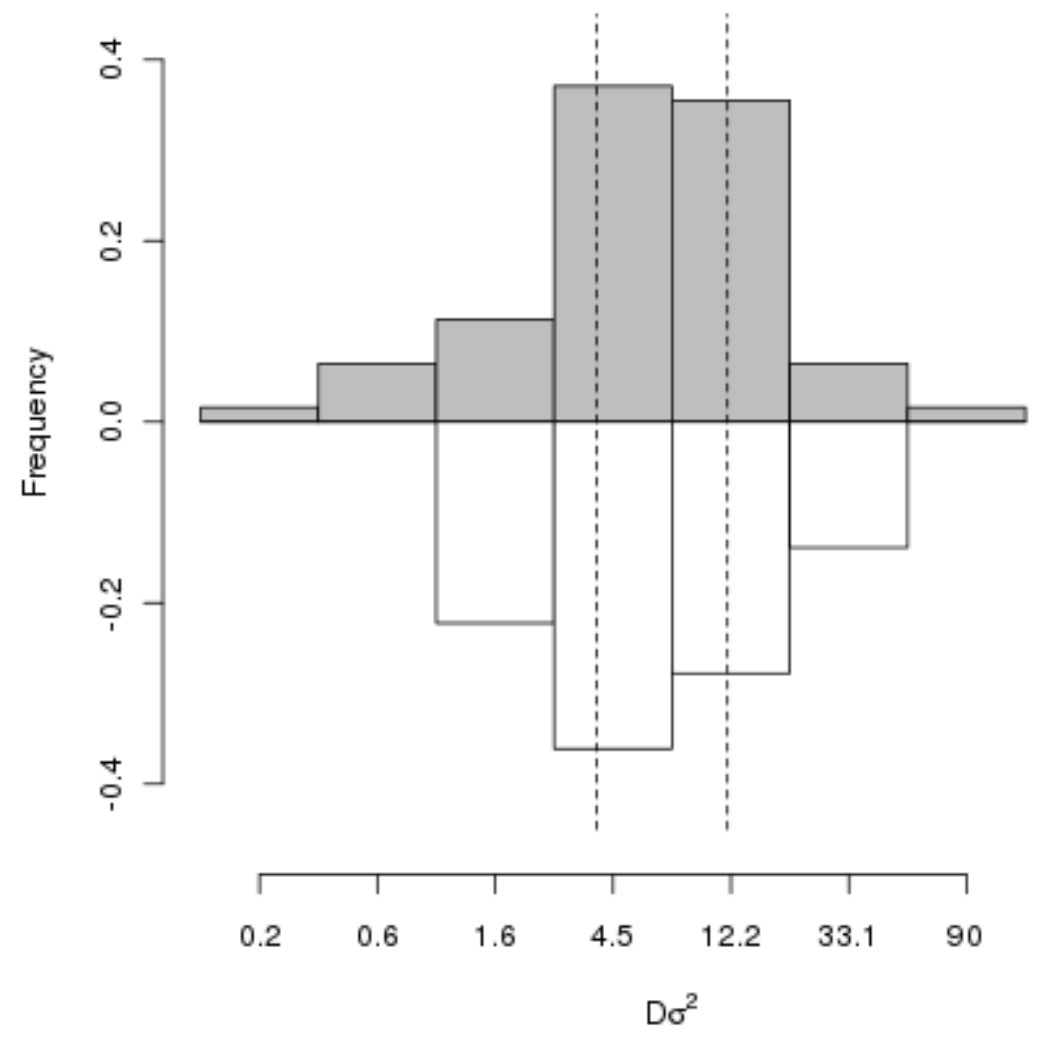


Fig. 2

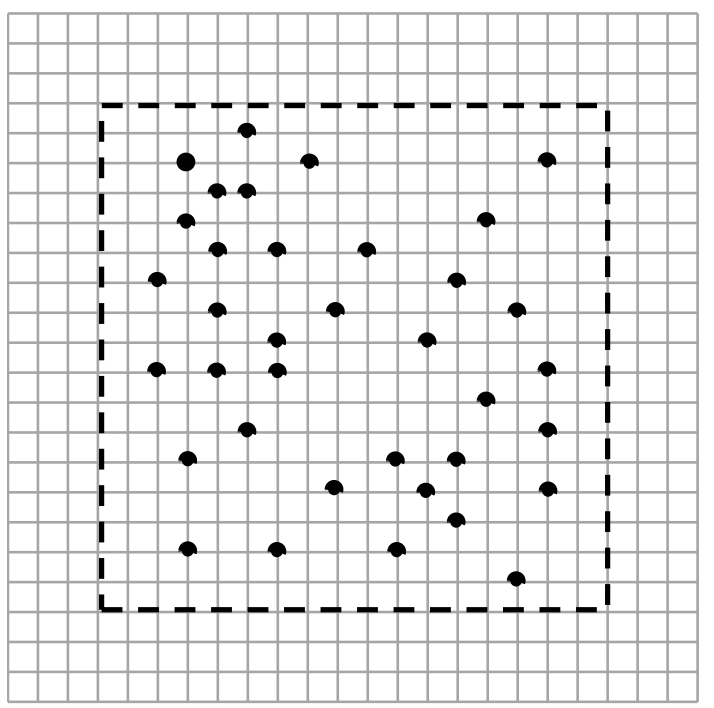

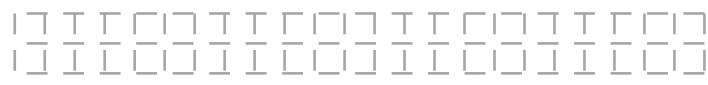

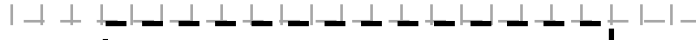
$1 \dashv+\mathbf{I}-|\dashv++\vdash|-|\dashv++|-|-|++\mathbf{I}|-|$

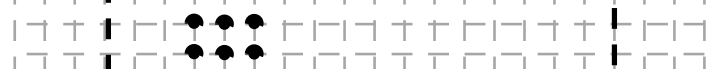

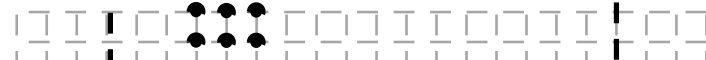

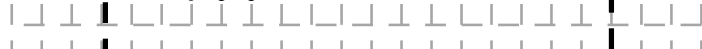
$|\dashv+\mathbf{I}|-|\dashv++\leftarrow|-|\dashv++|-|-|++-|-|-$

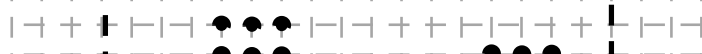

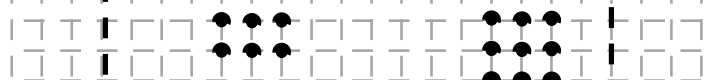

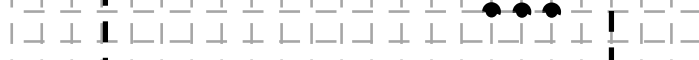
$|\dashv+\mathbf{I}|-|\dashv++\vdash|-|\dashv \bullet \bullet|-|++-|-\mid=$

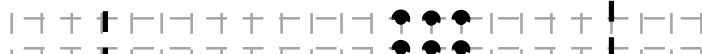

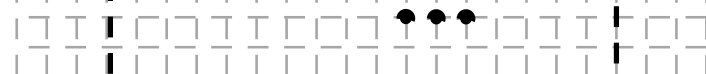
$1++$ L $1-++-1-1+-1-1++-1$

$|\dashv++|-|\dashv++\vdash|-|\dashv++\vdash|-|++\vdash|-\mid \dashv$

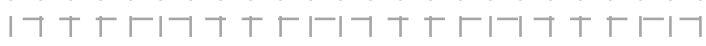


Fig. 3

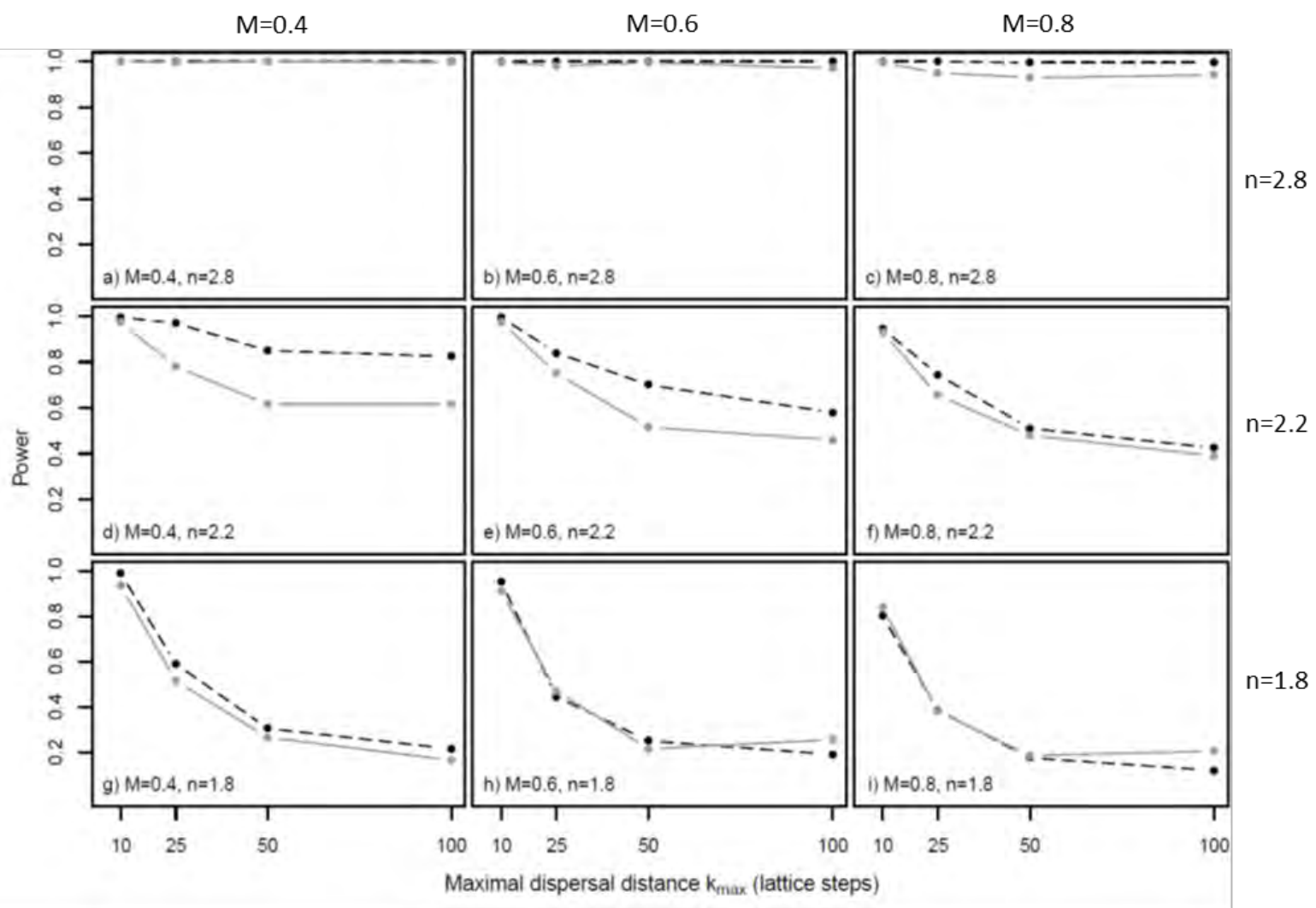


Fig. 4

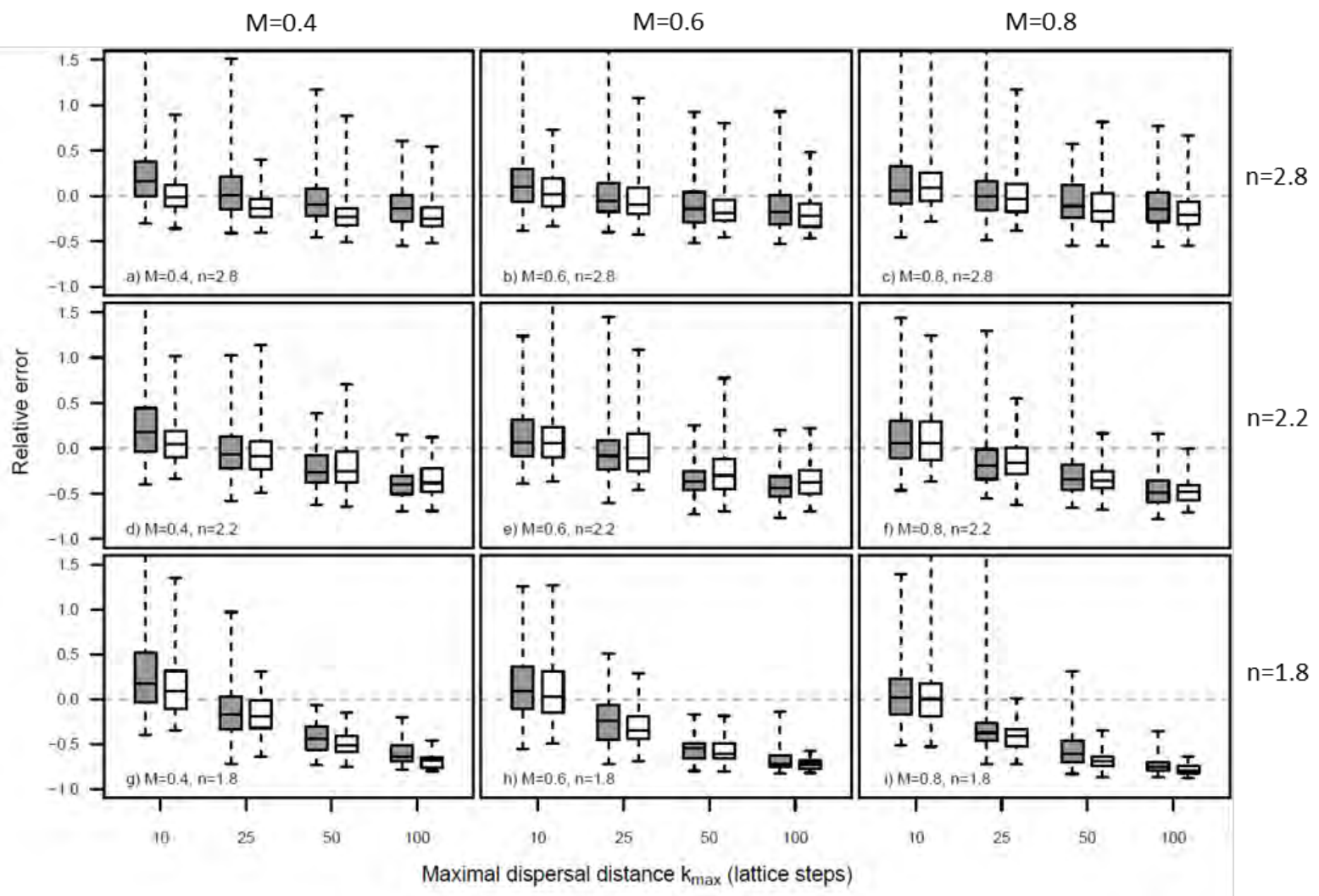




\section{Supplementary material}

Table S1: Literature survey of empirical $D \sigma^{2}$ values estimated from significant IBD patterns for animal and plant case studies that investigated two-dimensional spatial genetic structure (the product $D \sigma^{2}$ has a different scale in $1 D$ studies, Rousset 1997). These values were either taken directly from the papers, or calculated from related statistics, such as Sp (Vekemans\& Hardy 2004). When more than one value was available for a given species in a specific paper, only one was retained for drawing the histogram (see footnotes).

\begin{tabular}{|c|c|c|c|c|c|c|}
\hline Species & Taxon & Sampling unit & Density & Unit & $D \sigma^{2}$ & Reference \\
\hline Homo sapiens & Mammal & group & 24 & ind $/ \mathrm{km}^{2}$ & 17 & (Rousset 1997) \\
\hline Dipodomys spectabilis & Mammal & individual & 0.0002 & ind $/ \mathrm{m}^{2}$ & 2.6 & (Rousset 2000) \\
\hline Gnypetoscincus queenslandiae & Reptile & individual & 0.0136 & ind $/ \mathrm{m}^{2}$ & 6.7 & (Sumner et al. 2001) \\
\hline Chamaecrista fasciculata & Fabaceae & group & - & - & 10.7 & (Fenster et al. 2003) \\
\hline Ostrinia nubilalis & Insect & group & - & - & 4.9 & (Martel et al. 2003) \\
\hline Crassostrea virginica & Mollusc & group & 0.24 & ind $/ \mathrm{km}^{2}$ & 113.7 & (Rose et al. 2006) \\
\hline Martes americana & Mammal & individual & 0.46 & ind $/ \mathrm{km}^{2}$ & 6.6 & (Broquet et al. 2006) \\
\hline \multirow[t]{2}{*}{ Coenagrion mercuriale ${ }^{a}$} & Insect & individual & 0.0022 & ind $/ m^{2}$ & 30.7 & (Watts et al. 2007) \\
\hline & & group & 0.0023 & ind $/ \mathrm{m}^{2}$ & 31.3 & \\
\hline Plethodon cinereus & Amphibian & group & 2.82 & ind $/ m^{2}$ & 9.9 & (Cabe et al. 2007) \\
\hline Microtus arvalis & Mammal & individual & 1000 & ind $/ \mathrm{km}^{2}$ & 16.6 & (Gauffre et al. 2008) \\
\hline Bonasa bonasia & Bird & individual & 5.5 & ind $/ \mathrm{km}^{2}$ & 5 & (Sahlsten et al. 2008) \\
\hline Milicia excelsia ${ }^{b}$ & Moraceae (tree) & individual & 4.96 & ind $/ \mathrm{km}^{2}$ & 12.3 & (Bizoux et al. 2009) \\
\hline Corrallium rubrum ${ }^{c}$ & Cnidaria & group & - & - & 8 & (Ledoux et al. 2010) \\
\hline
\end{tabular}

(a) Based upon the data used to compare group-and individual-based approaches. (b) Computed from Table 2 for population Mindourou. (c) Computed from the slope value given in Fig. 2 for population Catalonia. 
Table S2: Simulation conditions. The parameters $M, n$, and $k_{\max }$ set the shape of the distribution of dispersal distances in the simulations. The values taken by these parameters result in a range of dispersal conditions characterized by $\sigma_{\text {sim }}^{2}$. The genetic structure observed at equilibrium is given by the mean pairwise genetic distance between sampled individuals $(a)$ or groups $\left(F_{\mathrm{ST}} /\left(1-F_{\mathrm{ST}}\right)\right.$ and the global $F_{\mathrm{ST}}$ averaged over 200 simulation replicates.

\begin{tabular}{|c|c|c|c|c|c|c|c|}
\hline \multirow{2}{*}{ Simulation } & \multirow{2}{*}{$M$} & \multirow{2}{*}{$n$} & \multirow{2}{*}{$K_{\max }$} & \multirow{2}{*}{$\sigma_{s i m}^{2}$} & \multicolumn{3}{|c|}{ mean genetic distance } \\
\hline & & & & & $a$ & $F_{\mathrm{ST}} /\left(1-F_{\mathrm{ST}}\right)$ & $F_{\mathrm{ST}}$ \\
\hline 1 & 0.4 & 2.8 & 10 & 1.12 & 0.202 & 0.103 & 0.093 \\
\hline 2 & 0.4 & 2.8 & 25 & 1.53 & 0.201 & 0.101 & 0.091 \\
\hline 3 & 0.4 & 2.8 & 50 & 1.83 & 0.203 & 0.103 & 0.093 \\
\hline 4 & 0.4 & 2.8 & 100 & 2.01 & 0.200 & 0.103 & 0.093 \\
\hline 5 & 0.4 & 2.2 & 10 & 1.99 & 0.159 & 0.071 & 0.066 \\
\hline 6 & 0.4 & 2.2 & 25 & 3.89 & 0.151 & 0.064 & 0.060 \\
\hline 7 & 0.4 & 2.2 & 50 & 6.03 & 0.151 & 0.063 & 0.059 \\
\hline 8 & 0.4 & 2.2 & 100 & 7.85 & 0.154 & 0.064 & 0.060 \\
\hline 9 & 0.4 & 1.8 & 10 & 3.08 & 0.133 & 0.050 & 0.047 \\
\hline 10 & 0.4 & 1.8 & 25 & 7.93 & 0.120 & 0.040 & 0.038 \\
\hline 11 & 0.4 & 1.8 & 50 & 15.23 & 0.115 & 0.037 & 0.036 \\
\hline 12 & 0.4 & 1.8 & 100 & 23.13 & 0.119 & 0.037 & 0.035 \\
\hline 13 & 0.6 & 2.8 & 10 & 1.68 & 0.101 & 0.074 & 0.068 \\
\hline 14 & 0.6 & 2.8 & 25 & 2.30 & 0.101 & 0.074 & 0.068 \\
\hline 15 & 0.6 & 2.8 & 50 & 2.75 & 0.104 & 0.075 & 0.069 \\
\hline 16 & 0.6 & 2.8 & 100 & 3.02 & 0.104 & 0.075 & 0.069 \\
\hline 17 & 0.6 & 2.2 & 10 & 2.99 & 0.071 & 0.051 & 0.048 \\
\hline 18 & 0.6 & 2.2 & 25 & 5.85 & 0.065 & 0.043 & 0.041 \\
\hline 19 & 0.6 & 2.2 & 50 & 9.08 & 0.063 & 0.042 & 0.040 \\
\hline 20 & 0.6 & 2.2 & 100 & 11.83 & 0.064 & 0.042 & 0.040 \\
\hline 21 & 0.6 & 1.8 & 10 & 4.63 & 0.050 & 0.035 & 0.034 \\
\hline 22 & 0.6 & 1.8 & 25 & 11.95 & 0.041 & 0.026 & 0.025 \\
\hline 23 & 0.6 & 1.8 & 50 & 22.99 & 0.038 & 0.023 & 0.022 \\
\hline 24 & 0.6 & 1.8 & 100 & 34.98 & 0.041 & 0.023 & 0.023 \\
\hline 25 & 0.8 & 2.8 & 10 & 2.24 & 0.077 & 0.057 & 0.054 \\
\hline 26 & 0.8 & 2.8 & 25 & 3.08 & 0.072 & 0.052 & 0.049 \\
\hline 27 & 0.8 & 2.8 & 50 & 3.68 & 0.075 & 0.053 & 0.050 \\
\hline 28 & 0.8 & 2.8 & 100 & 4.03 & 0.076 & 0.055 & 0.052 \\
\hline 29 & 0.8 & 2.2 & 10 & 4.00 & 0.047 & 0.036 & 0.034 \\
\hline 30 & 0.8 & 2.2 & 25 & 7.83 & 0.040 & 0.030 & 0.029 \\
\hline 31 & 0.8 & 2.2 & 50 & 12.16 & 0.039 & 0.028 & 0.027 \\
\hline 32 & 0.8 & 2.2 & 100 & 15.84 & 0.039 & 0.029 & 0.028 \\
\hline 33 & 0.8 & 1.8 & 10 & 6.20 & 0.031 & 0.024 & 0.023 \\
\hline 34 & 0.8 & 1.8 & 25 & 16.02 & 0.020 & 0.016 & 0.016 \\
\hline 35 & 0.8 & 1.8 & 50 & 30.86 & 0.018 & 0.015 & 0.015 \\
\hline 36 & 0.8 & 1.8 & 100 & 47.05 & 0.020 & 0.015 & 0.014 \\
\hline
\end{tabular}


Fig. S1 Mean pairwise genetic distance among individuals ( $\hat{a}$, in black) or groups $\left(F_{\mathrm{ST}} /\left(1-F_{\mathrm{ST}}\right)\right.$, in grey) averaged over all replicates of each simulation. The results are classified according to simulation conditions: the panels differ in values taken by $M$ and $n$, while the dots within each panel correspond to $k_{\max }=10,25,50$ and 100 , respectively. The x-axis gives the resulting $\sigma_{\text {sim }}^{2}$ averaged over all replicates of a simulation.

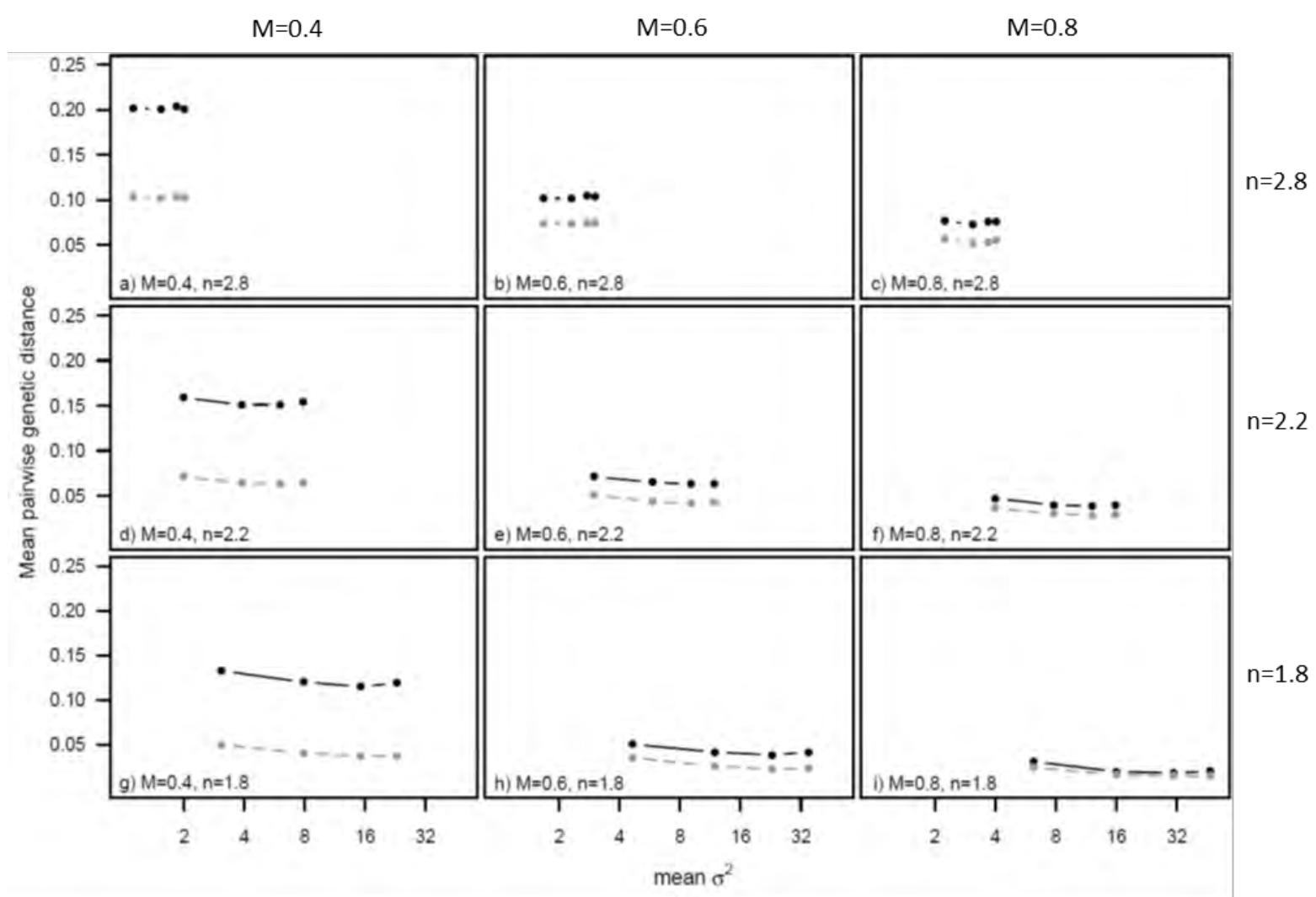


Fig. S2 -Coverage probability of $95 \%$ Confidence Intervals around $\sigma^{2}$ estimates from the regression of genetic- vs geographic distances between pairs of individuals (black) or groups (grey). Data from 200 replicates per simulation are shown (simulation conditions as in Figure S2).

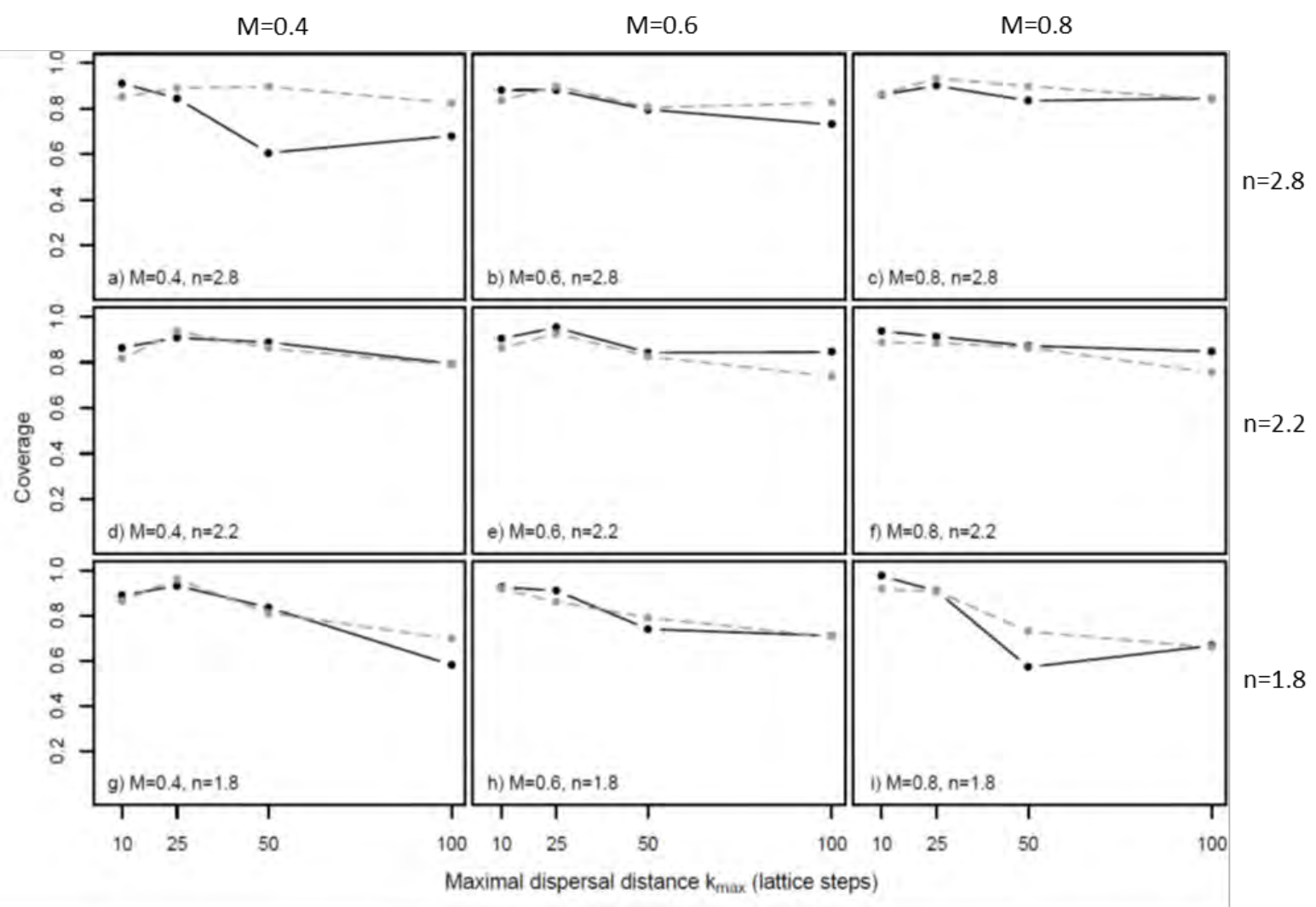


References for supplementary material

Bizoux JP, Dainou K, Bourland N, et al. (2009) Spatial genetic structure in Milicia excelsa (Moraceae) indicates extensive gene dispersal in a low-density wind-pollinated tropical tree. Molecular Ecology 18, 43984408.

Broquet T, Johnson CA, Petit E, et al. (2006) Dispersal and genetic structure in the American marten, Martes americana. Molecular Ecology 15, 1689-1697.

Cabe PR, Page RB, Hanlon TJ, et al. (2007) Fine-scale population differentiation and gene flow in a terrestrial salamander (Plethodon cinereus) living in continuous habitat. Heredity 98, 53-60.

Fenster CB, Vekemans X, Hardy O (2003) Quantifying gene flow from spatial genetic structure data in a metapopulation of Chamaecrista fasciculata (Leguminosae). Evolution 57, 995-1007.

Gauffre B, Estoup A, Bretagnolle V, Cosson JF (2008) Spatial genetic structure of a small rodent in a heterogeneous landscape. Molecular Ecology 17, 4619-4629.

Ledoux JB, Garrabou J, Bianchimani O, et al. (2010) Fine-scale genetic structure and inferences on population biology in the threatened Mediterranean red coral, Corallium rubrum. Molecular Ecology 19, 42044216.

Martel C, Rejasse A, Rousset F, Bethenod MT, Bourguet D (2003) Host-plant-associated genetic differentiation in Northern French populations of the European corn borer. Heredity 90, 141-149.

Rose CG, Paynter KT, Hare MP (2006) Isolation by distance in the eastern oyster, Crassostrea virginica, in Chesapeake Bay. Journal of Heredity 97, 158-170.

Rousset F (1997) Genetic differentiation and estimation of gene flow from F-statistics under isolation by distance. Genetics 145, 1219-1228.

Rousset F (2000) Genetic differentiation between individuals. Journal of Evolutionary Biology 13, 58-62.

Sahlsten J, Thorngren H, Hoglund J (2008) Inference of hazel grouse population structure using multilocus data: a landscape genetic approach. Heredity 101, 475-482. 
Sumner J, Rousset F, Estoup A, Moritz C (2001) Neighbourhood size, dispersal and density estimates in the prickly forest skink (Gnypetoscincus queens/andiae) using individual genetic and demographic methods. Molecular Ecology 10, 1917-1927.

Watts PC, Rousset F, Saccheri IJ, et al. (2007) Compatible genetic and ecological estimates of dispersal rates in insect (Coenagrion mercuriale : Odonata : Zygoptera) populations: analysis of 'neighbourhood size' using a more precise estimator. Molecular Ecology 16, 737-751. 